\title{
Análisis bibliométrico del balance vida/trabajo en mujeres médicas
}

\section{Analysis Bibliometric of Life Balance/Work in Medical Women}

\author{
Catalina Solano ${ }^{10}$ Beatriz Johanna Vega López ${ }^{2(-)}$ Adriana Chaves Parra ${ }^{3}$ Catalina Osorio Ospino ${ }^{4}$ \\ Lisset Fernanda Arroyo Malaver ${ }^{5}$ Luisana Castillo Carvajal ${ }^{6}$ Daisy Ximena Roa Saavedra ${ }^{70}$
}

1 Uróloga, Uroclin, Medellín, Antioquia, Colombia

2 Uróloga, Magister en epidemiología, Hospital Universitario de La Samaritana, Bogotá, Cundinamarca, Colombia

3 Uróloga, Uronorte, Cúcuta, Norte de Santander, Colombia

${ }^{4}$ Uróloga, Clinica comfamiliar, Pereira, Risaralda, Colombia

${ }^{5}$ Uróloga, Hospital Simón Bolívar, Bogotá, Cundinamarca, Colombia

6 Uróloga, Clínica Juan N corpas Bogotá, Cundinamarca, Colombia

7 Uróloga, Cooperativa Especializada de servicios urológicos del

Tolima. UROTOL, Ibagué, Tolima, Colombia

\begin{abstract}
Address for correspondence Beatriz Johanna Vega López, MD, MSc Dirección: Calle 140 \# 13-66, apartamento 1401, torre 3, Bogotá, DC 110121, Colombia (e-mail: drajohannavL@gmail.com).
\end{abstract}

Urol Colomb 2020;29:187-194.

\section{Resumen}

\section{Palabras clave}

- mujeres

- médico

- estilo de vida

- balance vida-trabajo

- estrés

- calidad de vida

- burnout profesional
Objetivo Realizar un análisis bibliométrico para conocer las tendencias de publicación en calidad de vida y balance vida/trabajo de mujeres médicas.

Métodos Se realizó un análisis bibliométrico retrospectivo de la literatura médica disponible desde 1996 hasta 2019 en la base de datos de MEDLINE, utilizando las herramientas estadísticas de FABUMED y PubReminer para la obtención de la información de las variables. Se utilizó el Journal Citation Reports 2017/2018 para el factor de impacto y el programa de software VOSviewer para el análisis de mapeo bibliométrico.

Resultados De un total de 204 referencias en 115 revistas, se presentó un crecimiento del 70,5\% de las publicaciones los últimos años. El país, revista e institución con mayor número de manuscritos fue Estados Unidos (22.5\%), Acad Med (4.9\%) con factor de impacto de 4,9 years Harvard medical School, respectivamente. El autor con mayor número de artículos fue Nomura K con 6 publicaciones y un HI de 21. El mapeo y análisis de las evoluciones de las palabras clave con respecto a la línea de tiempo, se centró principalmente en: balance vida -trabajo, sexismo, liderazgo mentoría, mujeres cirujanas. Conclusiones Las publicaciones sobre el tema balance vida/trabajo se han incrementado, permitiendo visualizar esta problemática y quizá lograr un impacto en el diseño de estrategias que permitan una mejor calidad de vida especialmente a las mujeres, quienes siguen teniendo una alta exigencia y responsabilidad en su vida familiar y a su vez, barreras profesionales e inequidad laboral. received

August 31, 2020

accepted

October 12, 2020
DOI https://doi.org/

$10.1055 / \mathrm{s}-0040-1721329$.

ISSN 0120-789X.

e ISSN 2027-0119.
Copyright () 2020, Thieme Revinter

Publicações Ltda.., Rua do Matoso 170 ,

Rio de Janeiro, RJ, CEP 20270-135, Brazil.

Todos los derechos reservados.

\section{License terms}

c) $(\oplus)$ 


\begin{abstract}
Keywords

- women

- doctor

- lifestyle

- life-work balance

- stress

- quality of life

- professional burnout

Objective Conduct a bibliometric analysis to learn about publication trends in quality of life and life balance/work of medical women.

Methods A retrospective bibliometric analysis of the medical literature available from 1996 to 2019 was performed in the MEDLINE database, using the statistical tools of FABUMED and PubReminer to obtain information on variables. The Journal Citation Reports 2017/2018 was used for the impact factor and the VOSviewer software program for bibliometric mapping analysis.

Results Out of a total of 204 references in 115 journals, there was a $70.5 \%$ growth in publications in recent years. The country, magazine and institution with the highest number of manuscripts was the United States (22.5\%), Acad Med (4.9\%) impact factor of 4.9 and Harvard medical School respectively. The author with the highest number of articles was Nomura $\mathrm{K}$ with 6 publications and a $\mathrm{HI}$ of 21. Mapping and analyzing the evolutions of keywords with respect to the timeline, focused mainly on: balance life work, sexism, mentoring leadership, women surgeons.

Conclusions Publications on the issue of life/work balance have increased, allowing to visualize this problem and perhaps achieve an impact on the design of strategies that allow a better quality of life especially for women, who continue to have a high demand and responsibility in their family life and in turn, professional barriers and inequity work.
\end{abstract}

\section{Introducción}

Actualmente el papel de la mujer en medicina y áreas científicas afines es indiscutible. Según estadísticas canadienses, las profesionales de la salud femeninas eran del $7 \%$ en el año 1961 years pasó a un 28\% para el año 2000, y así mismo sucedió con las mujeres estudiantes de medicina que llegaron a ser del 50\% a partir del año de $1995 .^{1,2}$ No obstante, a pesar de esta tendencia alentadora en un gremio tradicionalmente dominado por hombres, encontrar un equilibrio adecuado entre el trabajo y la vida diaria es un desafío particularmente mayor en las mujeres, ya que la sociedad les exige combinar con éxito el trabajo y los compromisos familiares de madres y esposas.

Es bien sabido que el gremio médico padece 1.5 veces más estrés comparado con otras áreas de la salud. ${ }^{3}$ Sin embargo, los factores estresantes adicionales a los que se enfrentan las mujeres médicas con respecto a los hombres (altas expectativas, múltiples roles, ambiente laboral, entre otros), las exponen a padecer con mayor frecuencia depresión, ansiedad, problemas maritales, y se han vinculado al aumento del consumo de alcohol, tabaco y desarrollo de trastornos somáticos secundarios. ${ }^{4}$ Este fenómeno ha llevado a la mayoría de mujeres médicas a escoger especialidades que les permitan tener mejor calidad de vida y mayor tiempo libre, con el objetivo de lograr un balance vida/ trabajo, aunque esto signifique sacrificar oportunidades que les posibiliten un desempeño profesional más exitoso. ${ }^{5,6}$

Del mismo modo, en la actualidad las mujeres médicas todavía perciben barreras de género dentro de ciertas especialidades, se sienten criticadas por tomar tiempo y permisos para su maternidad o actividades familiares y más grave aún, por dar a conocer en su entorno laboral signos de fatiga o estrés. ${ }^{7-9}$
Dado que la mujer es y será piedra angular en la familia y en la fuerza laboral actual, es indispensable entender los factores que generan impacto en su calidad de vida y que pueden llevar a una disminución en su rol como madres, esposas y profesionales; por tal razón, en el presente análisis bibliométrico se pretende realizar un acercamiento del balance vida/trabajo en las mujeres médicas, brindando una mejor comprensión de las tendencias de publicación acerca de este tema.

\section{Métodos}

Se realizó un análisis bibliométrico descriptivo y de corte retrospectivo de la literatura médica obtenida en MEDLINE a través de PUBMED, en relación con estilos de vida en mujeres médicas. Se utilizó la estrategia de búsqueda: "Physicians, Women" AND "work-life balance" AND (1996:2019[dp])). Se incluyeron todos los artículos con el fin de analizar toda la literatura médica indexada en relación con este tema desde 1996 hasta 2019.

Se utilizaron las herramientas estadísticas de FABUMED y PubReminer para la obtención de la información de las variables a analizar (número total de publicaciones y revistas, número y porcentaje de publicaciones por revista, país, idioma, artículos más citados, autores, instituciones involucradas, tipos de documentos y áreas de estudio).

Para la obtención del factor de impacto (FI), se usó el Journal Citation Reports 2017/2018 de las revistas encontradas para estimar la calidad de cada una de estas, ${ }^{10}$ y para el análisis de mapeo bibliométrico se utilizó el programa de software: VOSviewer versión 1.6.15, que explora las tendencias de palabras clave y los conceptos relacionados, así como mapas de redes de colaboración. Posterior a esto, se analizaron los resultados de forma 
descriptiva y con base en esta información, se elaboraron tablas y gráficas en Microsoft Office Excel 2017.

\section{Resultados}

\section{Datos Generales}

Se recuperaron un total de 204 referencias en 115 revistas (-Figura 1). Durante los 23 años de la revisión, la participación porcentual de la producción mundial de investigación fue de $14.7 \%$ (30/204) desde 1996 a 2008, 85.29\% (174/204) desde 2009 a 2019 (-Figura 2). Se presentó un crecimiento del $70.5 \%$ en las publicaciones de balance vida / trabajo en mujeres médicas consignadas en las bases de datos PubMed y FABUMED.

\section{Países}

La producción total de publicaciones se originó en 62 países (- Figura 3). De estos, el 49\% de las publicaciones se produjo en cinco países, liderado por Estados Unidos con 46 (22.5\%) publicaciones, seguido de Reino Unido con 27 (13.2\%), Japón 18 (8.8\%), Alemania 9 (4.9\%) y Canadá 6 (3.4\%). En Latinoamérica, Brasil ocupa el primer lugar con dos artículos (0.98\%), seguido de Argentina, Chile, Colombia, México, Uruguay y Venezuela con un artículo cada uno (0.49\%).

\section{Revistas}

Las 20 revistas descritas en la - Tabla 1, contienen el 37.2\% (76) de las publicaciones desde 1996-2019, el número de artículos publicados en cada una de éstas, el idioma predominantemente fue el inglés, y el FI para el año 2018 según el Journal Citation Reports. Del top 20 de las publicaciones, las cinco revistas con mayor porcentaje de publicaciones fueron: Acad Med 4.9\%, J Womens Health 3.9\%, Plastic Reconstr Surg 2.9\%, Nihon Geka Gakkai 2.4\% y J. Gen Intern Med 1.96. De estas 20 revistas sólo 2 de ellas tuvieron un FI mayor de 8 (Lancet y BMJ).

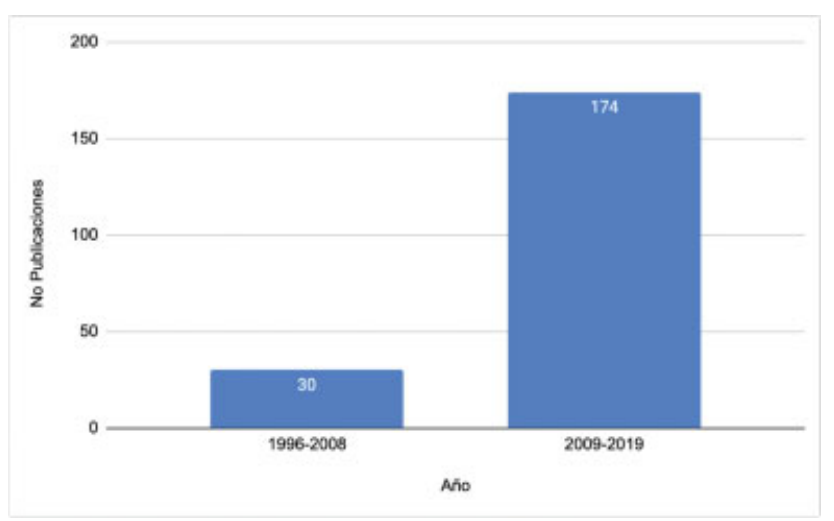

Fig. 2 Crecimiento del número de publicaciones desde 1996-2019 divididas por periodos.

\section{Tipos de documento y Áreas de estudio}

En el periodo comprendido entre 1996 a 2019 se observó que la mayoría de documentos fueron artículos de revista en un 78\% (-Figura 4), y las áreas de estudio que predominaron fueron medicina en un $72 \%$, seguida de ciencias sociales con $12.4 \%$ (-Figura 5).

\section{Artículos más citados}

Dentro de los diez artículos más citados, la publicación con mayor número de citaciones (220 en total), fue: Predictors of physician career satisfaction, work-life balance, and burnout, publicado en Obstetrics and Gynecology, revista con FI 5.5, cuya autora principal es una mujer Keeton, $K$ (h-index de 5 ). Los artículos con mayor número de citaciones provienen de Estados Unidos con el $70 \%$ y un pequeño porcentaje de Europa. Dentro de los temas a tratar, se dividen en las siguientes categorías: "burnout, mentors, female physician" (-Tabla 2).

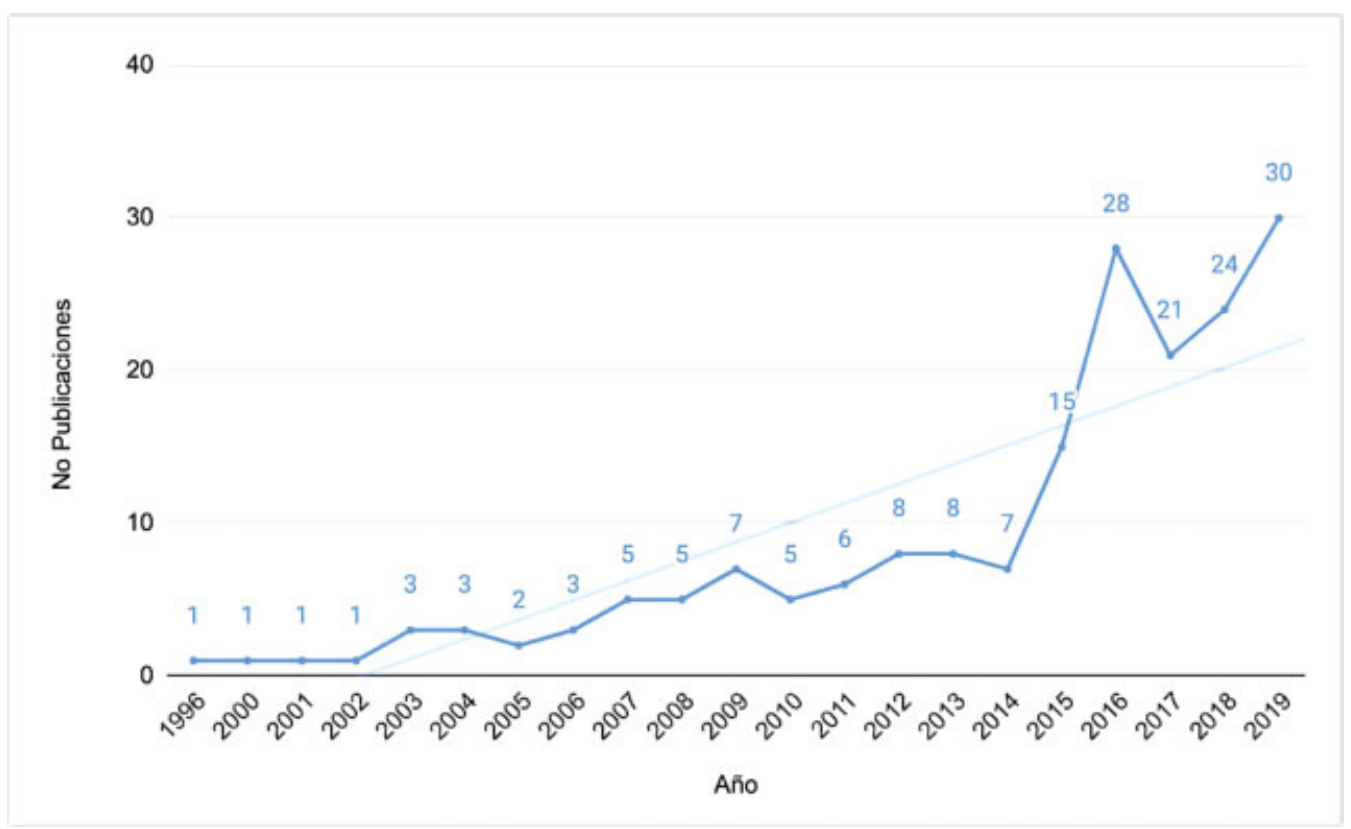

Fig. 1 Crecimiento de publicaciones globales en las últimas 3 décadas acerca de balance vida/trabajo en mujeres médicas. 


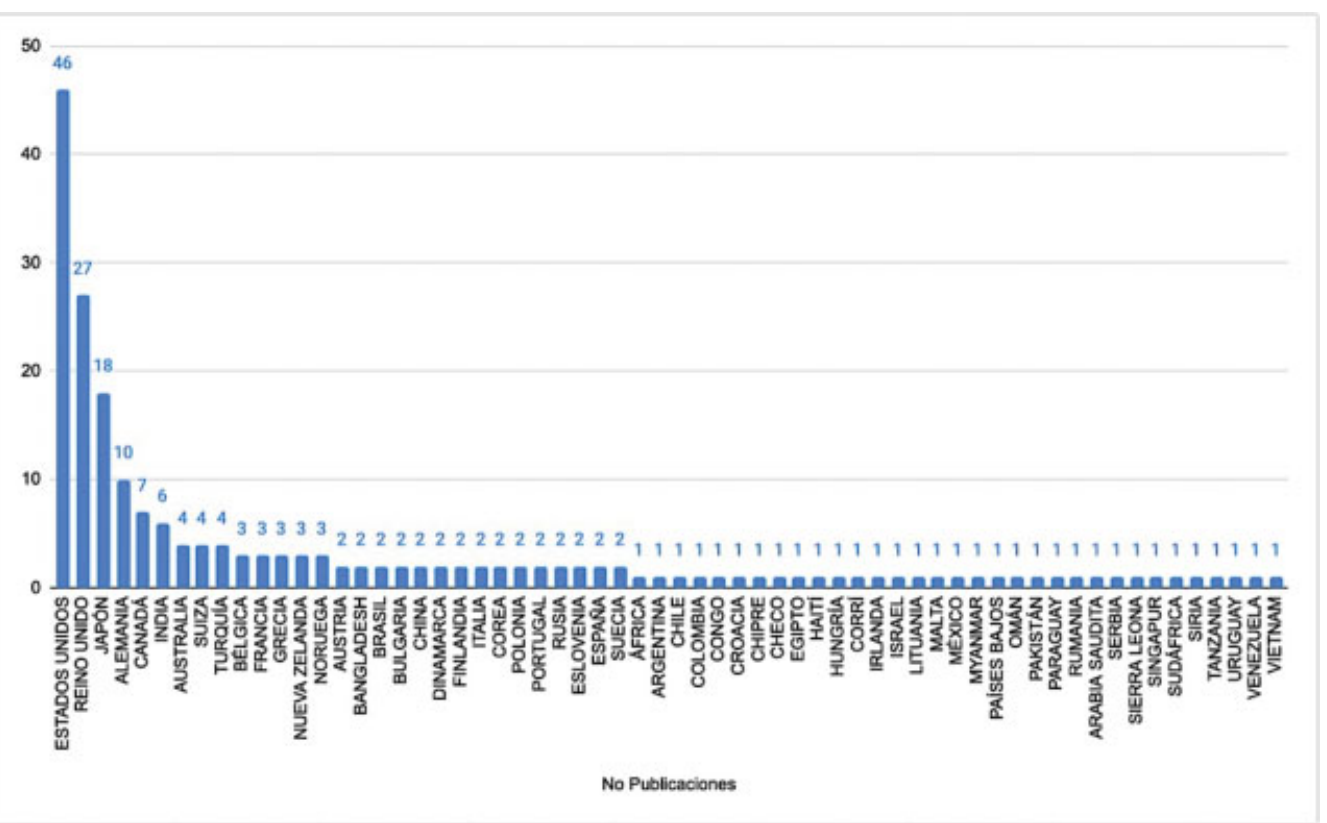

Fig. 3 Países participantes en las publicaciones 1996-2019.

\section{Autores}

En total se encontraron 1031 autores, de estos solamente tres presentaron más de cinco artículos publicados (-Tabla 3). El autor con mayor número de artículos es Nomura, K., de Graduate School of Medicine, con seis publicaciones y un HI de 21.

\section{Instituciones}

El top 10 de instituciones reportadas provienen de Estados Unidos; la que presentó mayor número de artículos acerca del tema fue Harvard medical School con 13 en total (- Figura 6).

Tabla 1 Top 20 de revistas con publicación sobre balance vida/trabajo en mujeres médicas en el período 1996 a 2019

\begin{tabular}{|c|c|c|c|c|}
\hline Revista & Artículos & $\begin{array}{l}\text { Factor de impacto } \\
2017-2018\end{array}$ & Idioma & País \\
\hline Acad Med & 10 & 4.93 & Inglés & Estados Unidos \\
\hline J Womens Health (Larchmt) & 8 & * & $*$ & * \\
\hline Plast Reconstr Surg & 6 & 3.94 & Inglés & Estados Unidos \\
\hline Nihon Geka Gakkai Zasshi & 5 & 0.55 & Japonés & Japón \\
\hline J Gen Intern Med & 4 & 4.60 & Inglés & Estados Unidos \\
\hline J R Soc Med & 4 & * & Inglés & Inglaterra \\
\hline Pediatrics & 4 & 4.6 & Inglés & Estados Unidos \\
\hline Tohoku J Exp Med & 4 & 1.58 & Inglés & Japón \\
\hline World J Surg & 4 & 2.76 & Inglés & Estados Unidos \\
\hline BMC Health Serv Res & 3 & 1.93 & Inglés & Inglaterra \\
\hline BMC Med Educ & 3 & 1.87 & Inglés & Inglaterra \\
\hline GMS Z Med Ausbild & 3 & * & * & * \\
\hline Lancet & 3 & 59.10 & Inglés & Inglaterra \\
\hline Tidsskr Nor Laegeforen & 3 & $*$ & $*$ & * \\
\hline Arch Gynecol Obstet & 2 & 2.19 & Inglés & Alemania \\
\hline BMJ & 2 & 27.6 & Inglés & Inglaterra \\
\hline BMJ Open & 2 & 2.37 & Inglés & Inglaterra \\
\hline Can Fam Physician & 2 & 2.18 & Multilenguaje & Canadá \\
\hline Clin Orthop Relat Res & 2 & 4.15 & Inglés & Estados Unidos \\
\hline Eur J Pediatr Surg & 2 & 1.14 & Inglés & Alemania \\
\hline
\end{tabular}




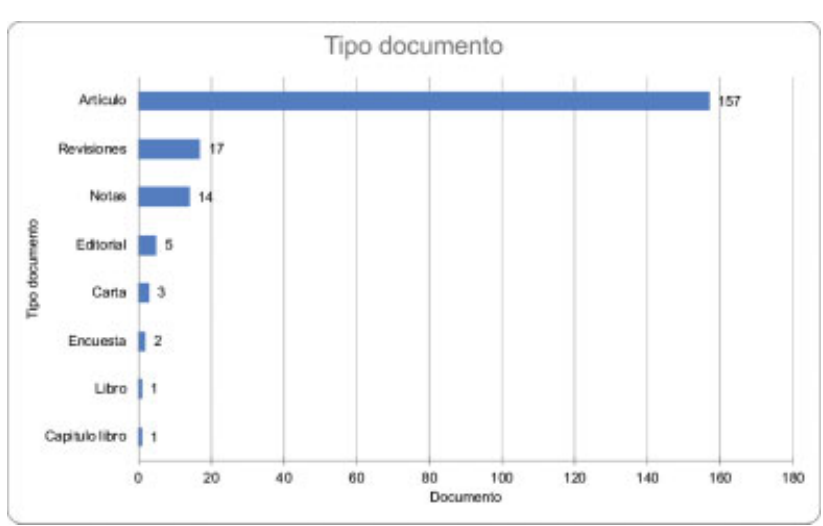

Fig. 4 Porcentajes de categorías de documento desde 1996-2019.

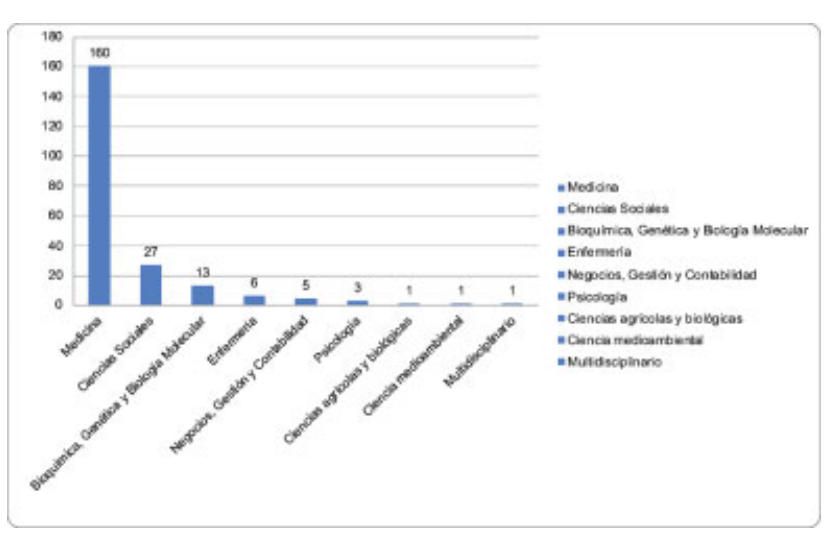

Fig. 5 Porcentaje de áreas de estudio.

\section{Análisis de co-ocurrencia utilizando palabras claves}

Utilizando la herramienta VOSviewer se analizaron los 204 artículos, identificando las áreas de investigación mas relevantes del tema. Se evaluó el número de co-ocurrencia entre palabras clave y palabras clave por título y resumen. El análisis de co-ocurrencia realizado con la unidad de análisis de las palabras clave con un mínimo de 5 veces en las repeticiones, se encontraron 91 palabras organizadas en cinco grupos o clusters (-Figura 7a).

El Grupo 1 (rojo) hace referencia al balance familia trabajo, en relación con las palabras clave: carga laboral, estrés, burnout, calidad de vida. El Grupo 2 (verde), se basa principalmente en las mujeres médicas, la rotación en puestos de trabajo, sexismo, y liderazgo. Por último, en el Grupo 3 (azul) predomina la correlación de las palabras clave que existe entre: mujeres, mentoría, balance vida trabajo, estilos de vida. ( - Figura 7 a).

El mapeo y análisis de las evoluciones de las palabras clave con respecto a la línea de tiempo, permite identificar la evolución de la literatura sobre el tema estudiado. Se evidenció que antes de 2014 los temas más predominantes eran: la fuerza de trabajo, su relación con la carga laboral y escogencia de especialidades; y en los últimos años, los tópicos se centraron más en: balance vida -trabajo, sexismo, liderazgo mentoría, mujeres cirujanas (-Figura 7b).

\section{Análisis de co-ocurrencia de las palabras clave en títulos y resúmenes}

En cuanto a las palabras con mayor número de co-ocurrencia en relación con los títulos y resúmenes, se encontraron 184 , con un mínimo de cinco veces, siendo la más relevante lifebalance (-Tabla 4).

Tabla 2 Top diez de los artículos más citados en sobre balance vida/trabajo en mujeres médicas durante el período 1996 a 2019 :

\begin{tabular}{|c|c|c|c|c|c|c|c|}
\hline Posición & Tópico & Titulo & Primer autor & Revista & Año & $\begin{array}{l}\text { Número de } \\
\text { citaciones }\end{array}$ & País \\
\hline 1 & $\begin{array}{l}\text { Burnout | } \\
\text { Depersonalization | } \\
\text { Medical Students }\end{array}$ & $\begin{array}{l}\text { Predictors of physician } \\
\text { career satisfaction, work- } \\
\text { life balance, and burnout }\end{array}$ & Keeton, K. & $\begin{array}{l}\text { Obstetrics and } \\
\text { Gynecology. }\end{array}$ & 2007 & 220 & $\begin{array}{l}\text { Estados } \\
\text { Unidos }\end{array}$ \\
\hline 2 & $\begin{array}{l}\text { Burnout I } \\
\text { Depersonalization | } \\
\text { Medical Students }\end{array}$ & $\begin{array}{l}\text { Physician satisfaction and } \\
\text { burnout at different } \\
\text { career stages }\end{array}$ & Dyrbye, L.N. & $\begin{array}{l}\text { Mayo Clinic } \\
\text { Proceedings. }\end{array}$ & 2013 & 202 & $\begin{array}{l}\text { Estados } \\
\text { Unidos }\end{array}$ \\
\hline 3 & $\begin{array}{l}\text { Academic Medicine I } \\
\text { Mentors I Female } \\
\text { Physician }\end{array}$ & $\begin{array}{l}\text { Inadequate Progress for } \\
\text { Women in Academic } \\
\text { Medicine: Findings from } \\
\text { the National Faculty Study }\end{array}$ & Carr, P.L. & $\begin{array}{l}\text { Journal of } \\
\text { Women's Health. }\end{array}$ & 2015 & 118 & $\begin{array}{l}\text { Estados } \\
\text { Unidos }\end{array}$ \\
\hline 4 & $\begin{array}{l}\text { Academic Medicine I } \\
\text { Mentors I Female } \\
\text { Physician }\end{array}$ & $\begin{array}{l}\text { The impact of gender and } \\
\text { parenthood on } \\
\text { physicians' careers - } \\
\text { Professional and personal } \\
\text { situation seven years after } \\
\text { graduation }\end{array}$ & $\begin{array}{l}\text { Buddeberg- } \\
\text { Fischer, B. }\end{array}$ & $\begin{array}{l}\text { BMC Health } \\
\text { Services Research. }\end{array}$ & 2010 & 102 & Suiza \\
\hline 5 & $\begin{array}{l}\text { Academic Medicine I } \\
\text { Mentors I Female } \\
\text { Physician }\end{array}$ & $\begin{array}{l}\text { Why do women choose or } \\
\text { reject careers in academic } \\
\text { medicine? A narrative } \\
\text { review of empirical } \\
\text { evidence }\end{array}$ & $\begin{array}{l}\text { Edmunds, L. } \\
\text { D. }\end{array}$ & The Lancet. & 2016 & 99 & $\begin{array}{l}\text { Reino } \\
\text { Unido }\end{array}$ \\
\hline 6 & $\begin{array}{l}\text { Academic Medicine I } \\
\text { Mentors | Female } \\
\text { Physician }\end{array}$ & $\begin{array}{l}\text { Stories from early-career } \\
\text { women physicians who } \\
\text { have left academic }\end{array}$ & Levine, R.B. & $\begin{array}{l}\text { Academic } \\
\text { Medicine. }\end{array}$ & 2011 & 89 & $\begin{array}{l}\text { Estados } \\
\text { Unidos }\end{array}$ \\
\hline
\end{tabular}


Tabla 2 (Continued)

\begin{tabular}{|c|c|c|c|c|c|c|c|}
\hline Posición & Tópico & Titulo & Primer autor & Revista & Año & $\begin{array}{l}\text { Número de } \\
\text { citaciones }\end{array}$ & País \\
\hline & & $\begin{array}{l}\text { medicine: A qualitative } \\
\text { study at a single } \\
\text { institution }\end{array}$ & & & & & \\
\hline 7 & $\begin{array}{l}\text { Academic Medicine I } \\
\text { Mentors I Female } \\
\text { Physician }\end{array}$ & $\begin{array}{l}\text { Organizational climate } \\
\text { and family life: How these } \\
\text { factors affect the status of } \\
\text { women faculty at one } \\
\text { medical school }\end{array}$ & Shollen, S.L. & $\begin{array}{l}\text { Academic } \\
\text { Medicine. }\end{array}$ & 2009 & 87 & $\begin{array}{l}\text { Estados } \\
\text { Unidos }\end{array}$ \\
\hline 8 & $\begin{array}{l}\text { Self-Employment I } \\
\text { Vocational } \\
\text { Rehabilitation I } \\
\text { Physical Disability }\end{array}$ & $\begin{array}{l}\text { The generation and } \\
\text { gender shifts in medicine: } \\
\text { An exploratory survey of } \\
\text { internal medicine } \\
\text { physicians }\end{array}$ & Jovic, E. & $\begin{array}{l}\text { BMC Health } \\
\text { Services Research }\end{array}$ & 2006 & 84 & Canadá \\
\hline 9 & $\begin{array}{l}\text { Burnout | } \\
\text { Depersonalization | } \\
\text { Medical Students }\end{array}$ & $\begin{array}{l}\text { Predictors and Outcomes } \\
\text { of Burnout in Primary } \\
\text { Care Physicians. }\end{array}$ & Rabatin, J. & $\begin{array}{l}\text { Journal of Primary } \\
\text { Care \& } \\
\text { Community } \\
\text { Health }\end{array}$ & 2016 & 71 & $\begin{array}{l}\text { Estados } \\
\text { Unidos }\end{array}$ \\
\hline 10 & $\begin{array}{l}\text { Burnout I } \\
\text { Depersonalization | } \\
\text { Medical Students }\end{array}$ & $\begin{array}{l}\text { Satisfaction with work-life } \\
\text { balance and the career } \\
\text { and retirement plans of } \\
\text { US oncologists }\end{array}$ & $\begin{array}{l}\text { Shanafelt, T. } \\
\text { D. }\end{array}$ & $\begin{array}{l}\text { Journal of Clinical } \\
\text { Oncology }\end{array}$ & 2014 & 70 & $\begin{array}{l}\text { Estados } \\
\text { Unidos }\end{array}$ \\
\hline
\end{tabular}

Tabla 3 Los diez autores principales con número de citaciones, h-index, institución y país:

\begin{tabular}{|l|l|l|l|l|}
\hline Autor & \# de artículos & h-index & Institución & País \\
\hline Nomura, K. & 6 & 21 & Graduate School of Medicine. & Japón \\
\hline Okinaga, H. & 6 & 10 & Support Center for women Physicians and Researchers. & Japón \\
\hline Goldacre, M.J. & 5 & 60 & University of Oxford Medical Sciences Division & Reino Unido \\
\hline Buddeberg-Fischer, B. & 4 & 24 & Rehabilitation Clinic Seewis. & Suiza \\
\hline Hiraike, H. & 4 & 12 & Teikyo University. & Japón \\
\hline Jagsi, R. & 4 & 54 & University of Michigan Medical School. & Estados Unidos \\
\hline Kawase, K & 4 & 10 & The Jikei University School of Medicine. & Japón \\
\hline Klaghofer, R. & 4 & 40 & Universitatsspital Zurich. & Suiza \\
\hline Lambert, T.W. & 4 & 31 & University of Oxford Medical Sciences Division. & Reino Unido \\
\hline Shanafelt, T.D. & 4 & 85 & Stanford University. & Estados Unidos \\
\hline
\end{tabular}

\section{Discusión}

La importancia de la mujer en la sociedad, las altas expectativas, los múltiples roles y el ambiente laboral, se han identificado como fuentes principales de estrés ${ }^{2}$ y especialmente en las mujeres médicas para lograr el

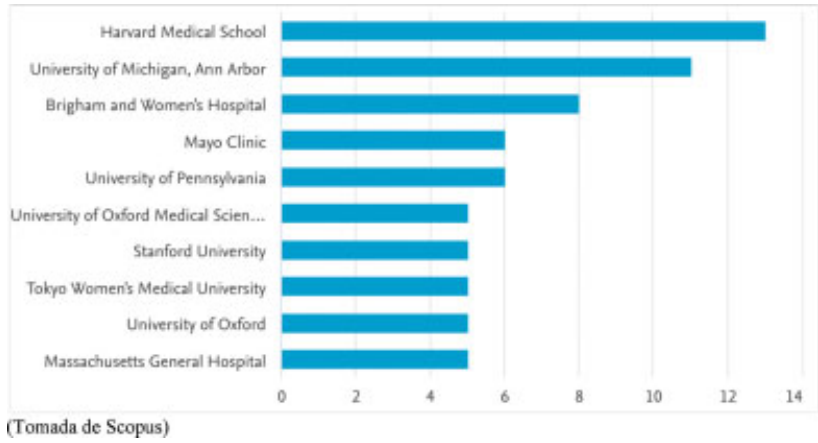

Fig. 6 Las diez principales Instituciones.

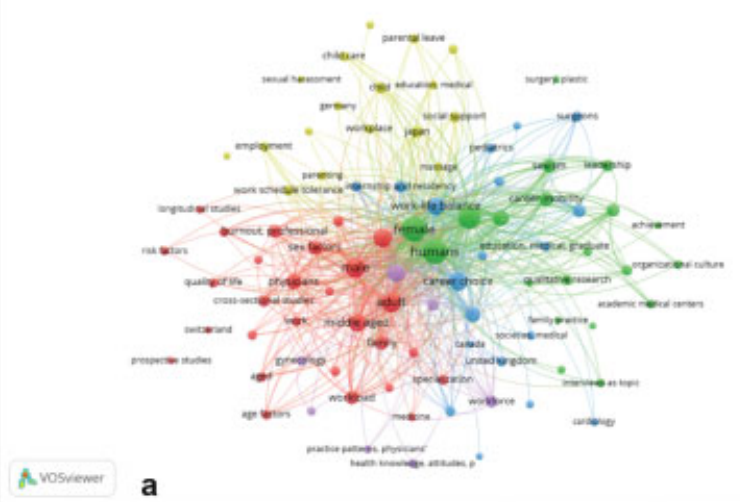

Fig. 7 (a) Análisis de co-ocurrencia de palabras clave en los estudios de balance vida/trabajo en mujeres medicas. (b) Mapeo y agrupamiento de las palabras claves en línea de tiempo entre 20102018. 
Tabla 4 Co-ocurrencia de las palabras clave en títulos y resúmenes:

\begin{tabular}{|l|l|l|}
\hline Término & Ocurrencia & Relevancia \\
\hline Life-balance & 110 & 0.04 \\
\hline Hour & 45 & 0.53 \\
\hline Choice & 28 & 0.24 \\
\hline Surgery & 27 & 0.54 \\
\hline Surgeon & 26 & 0.69 \\
\hline Theme & 23 & 0.80 \\
\hline Lack & 23 & 0.35 \\
\hline Burnout & 22 & 1.49 \\
\hline Academic medicine & 22 & 1.13 \\
\hline Female physician & 21 & 0.79 \\
\hline Environment & 20 & 0.37 \\
\hline
\end{tabular}

equilibrio entre el trabajo y la familia o vida personal, debido la demanda en el entrenamiento, la disponibilidad de tiempo laboral y las múltiples responsabilidades. ${ }^{11}$

Como se señala en la revisión de Robinson y col., el $26.4 \%$ de las médicas presentan un fuerte conflicto de rol comparado con el $6.1 \%$ de los médicos, y esto debido a que históricamente se acepta en la sociedad, que en los hombres las necesidades del trabajo están primero que las necesidades familiares. ${ }^{12}$ Este desequilibrio vida/trabajo lleva finalmente a padecer con mayor frecuencia estrés, que a largo plazo puede provocar agotamiento, depresión, insomnio, problemas físicos y enfermedades. ${ }^{13}$

Todo lo anterior promueve la búsqueda del balance vida/ trabajo en las médicas y con ello la publicación de investigaciones con esta premisa, como quedó demostrado en el presente estudio con el análisis de co-ocurrencia entre las palabras clave y palabras por título y resumen, destacando la relación con carga laboral, estrés, burnout y calidad de vida.

Las publicaciones presentaron un incremento a partir del año 2008, con un crecimiento global del 14.2\%, sin embargo, todos estos datos pueden no ser tan exactos, ya que en la literatura la información brindada es escasa y reciente. Este aumento, podría explicarse por diferentes factores como lo es el mayor ingreso de las mujeres en el ejercicio médico, donde en el 2010 era del 30\% comparado con el $8 \%$ en 1970. ${ }^{14}$

En relación con el FI, este fue relativamente bajo para la mayoría de las revistas donde el 35\% de las primeras 20, tienen un FI superior a tres, y solo el $10 \%$ tiene un valor superior a 8. Por otro lado, las publicaciones se han hecho principalmente en Estados Unidos, y dentro del bajo porcentaje proveniente de Latinoamérica, se originan esencialmente en Brasil. La categoría de las publicaciones más importante fue la de los artículos originales y especialmente en el área de medicina, procedentes la gran mayoría de Estados Unidos (70\%), de Instituciones representativas como Harvard Medical School, University of Michigan, Brigham and Women's Hospital, Mayo Clinic y University of Pennsylvania.

Las limitaciones de los análisis bibliométricos, tienen sesgos de lenguaje, sesgos en el país de autor, solo se nombra autores principales, afectando artículos con colaboración multinacional y co-autoria, las citaciones de los artículos las cuales son más predominantes en artículos más antiguos, no solamente se basa en la calidad de ellos. Finalmente, en la búsqueda se pueden encontrarla artículos relacionados a otros tópicos y no precisamente a los criterios de búsqueda.

A la fecha no existen estudios bibliométricos publicados con respecto a este tema, por lo que la comparación con los resultados del presente estudio está limitada al igual que la aplicabilidad a nuestra sociedad. Cabe resaltar que las metodologías de la mayoría de las investigaciones son cualitativas y proporcionan una visiónn de la problemática de los múltiples roles de las mujeres en la sociedad. ${ }^{6}$

El presente análisis bibliométrico incentiva a realizar publicaciones científicas futuras sobre el tema y generar literatura colombiana y latinoamericana que pueda ser aplicable, y tenga impacto en la sociedad al aportar conocimiento de las expectativas profesionales, rol laboral y familiar de las médicas y así disminuir el efecto del estrés y lograr el balance vida/trabajo en dicha población.

\section{Conclusiones}

En los últimos años gracias al mayor impacto que ha tenido la mujer en el campo laboral y especialmente en medicina, paralelamente ha comprometido su calidad de vida por cuenta del desequilibrio generado entre un rol profesional cada vez más demandante versus su rol familiar y sus responsabilidades e intereses personales.

Las publicaciones sobre el tema se han incrementado los últimos 10 años, lo cual ha permitido visibilizar esta problemática logrando que las instituciones diseñen e implementen mejoras que se traduzcan en un aumento en calidad de vida para sus empleados, especialmente a las mujeres quienes siguen teniendo una alta exigencia y responsabilidad en su vida familiar y a su vez, barreras profesionales e inequidad laboral.

Conflicto de intereses

Los autores declaran no tener ningún conflicto de intereses.

\section{Referencias}

1 Mobilos S, Chan M, Brown JB. Women in medicine: the challenge of finding balance. Can Fam Physician 2008;54(09):1285-1286.e5

2 Kass RB, Souba WW, Thorndyke LE. Challenges confronting female surgical leaders: overcoming the barriers. J Surg Res 2006;132(02):179-187

3 Belkić K, Nedic O. Workplace stressors and lifestyle-related cancer risk factors among female physicians: assessment using the Occupational Stress Index. J Occup Health 2007;49(01):61-71

4 Bergman B, Ahmad F, Stewart DE. Physician health, stress and gender at a university hospital. J Psychosom Res 2003;54(02): $171-178$ 
5 Dorsey ER, Jarjoura D, Rutecki GW. Influence of controllable lifestyle on recent trends in specialty choice by US medical students. JAMA 2003;290(09):1173-1178

6 Schueller-Weidekamm C, Kautzky-Willer A. Challenges of worklife balance for women physicians/mothers working in leadership positions. Gend Med 2012;9(04):244-250

7 Lambert EM, Holmboe ES. The relationship between specialty choice and gender of U.S. medical students, 1990-2003. Acad Med 2005;80(09):797-802

8 Sinden NJ, Sherriff JM, Westmore SE, Greenfield SM, Allan TF. Patterns of child-bearing behaviour amongst female hospital doctors and GPs. Fam Pract 2003;20(04):486-488

9 Brown AJ, Swinyard W, Ogle J. Women in academic medicine: a report of focus groups and questionnaires, with conjoint analysis. J Womens Health (Larchmt) 2003;12(10):999-1008
10 SJR Scientific Journal Rankings [Internet]. [cited 2020 Aug 30] Available from: https://www.scimagojr.com/journalrank.php? country $=\mathrm{CO}$

11 Golik M. Las expectativas de equilibrio entre vida laboral y vida privada y las elecciones laborales de la nueva generación. Cuad Am 2013;25(46):107-133

12 Robinson GE. Stresses on women physicians: consequences and coping techniques. Depress Anxiety 2003;17(03):180189

13 Petek D, Gajsek T, Petek Ster M. Work-family balance by women GP specialist trainees in Slovenia: a qualitative study. BMC Med Educ 2016;16:31

14 Stewart DE, Ahmad F, Cheung AM, Bergman B, Dell DL. Women physicians and stress. J Womens Health Gend Based Med 2000;9 (02):185-190 